Vietnam Academy of Science and Technology
(VAST)

\title{
Improvement of the accuracy of the quasigeoid model VIGAC2017
}

\author{
Ha Minh Hoa \\ Vietnam Institute of Geodesy and Cartography (VIGAC)
}

Received 14 June 2017; Received in revised form 25 October 2017; Accepted 10 November 2017

\begin{abstract}
A national spatial reference system will be constructed based on a highly accurate national quasigeoid model with accuracy more than $4 \mathrm{~cm}$. In Vietnam at the present stage there isn't a detailed gravimetric measurement in mountainous regions and marine area. So with the purpose of improvement of accuracy of the national quasigeoid model VIGAC2017, we only can solve the task of fitting this model to national quasigeoid heights obtained from heights GPS/first, second orders levelling quasigeoid heights through least squares collocation.

This scientific article will introduce a first research result for improvement of accuracy of the quasigeoid model VIGAC2017 on the base of it's fitting to 194 national quasigeoid heights by the least squares collocation. Research results show that accuracy of the quasigeoid model VIGAC2017 will be obtained at level of $\pm 0,058 \mathrm{~m}$ and increased to $20,69 \%$.
\end{abstract}

Keywords: National spatial reference system; national quasigeoid height; least squares collocation; covariance matrix; semivariogram; semivariance function.

(C2018 Vietnam Academy of Science and Technology

\section{Introduction}

A wide application of GNSS technology with GNSS data processing in ITRF and a combined usage of detailed gravimetric data and more accurate with every passing day Earth Gravity Model (EGM) for the construction of a highly accurate national quasigeoid model naturely lead to a bulding of a national spatial reference system. Ha Minh Hoa, 2017 had found that the most impotant base for the bulding of the national spatial reference system is the national quasigeoid model with ac-

*Corresponding author, Email: minhhoavigac@gmail,com curacy more than $\pm 4 \mathrm{~cm}$, which is the guarantee that the national geodetic height of every point on the national territory is equal to the sum of the it's national normal height and national quasigeoid height.

At present, many countries had constructed the highly accurate national quasigeoid/geoid models, for example, OSGM2002 (United Kingdom) with accuracy at level $\pm 3,2 \mathrm{~cm}$ (Iliffe J.C., Ziebart M,, Cross P.A., Forsberg R., Strykowski G., Tscherning C.C., 2003), USGG2009 (United States) with accuracy at level \pm (3-4) cm (Roman D. R., Y.M. Wang, J. Saleh, X. Li, 2010), CGG2013 (Canada) with accuracy more $\pm 3 \mathrm{~cm}$ on the $80 \%$ 
continent part (Huang J., Véronneau M., 2013), GCG16 (Germany) with accuracy more $\pm 1 \mathrm{~cm}$ (Alps max $2 \mathrm{~cm}$, marine area 2-6 cm) (Quasigeoid of the Federal Republic of Germany GCG2016).

The fit of gravimetric geoid/quasigeoid model to GPS/levelling geoid/quasigeoid heights through the least squares collocation had been accomplished in many countries. For example, the geoid model OSGM2002 had been fitted to the $179 \mathrm{GPS} /$ levelling geoid heights $\mathrm{cm}$ (Iliffe J.C., Ziebart M., Cross P.A., Forsberg R., Strykowski G., Tscherning C.C., 2003). In (Metin Soycan, 2014) had been presented results of fitting EGM2008 derived geoid heights to the $87 \mathrm{GPS} /$ leveling geoid heights in Turkey.

(Ha Minh Hoa, 2017) has presented results of construction of the initial national spatial referense system on base of orientation of the WGS84 ellipsoid to best fit it to the Hon Dau local quasigeoid at tide gauge Hon Dau with using the most stable 164 co located GPS observations first and second orders bench marks. When the national quasigeoid heights $\zeta$ have been calculated from the GPS/first and second orders levelling quasigeoid heights $\zeta_{G P S}$ / leveling by formula:

$$
\zeta=\zeta_{G P S / \text { leveling }}+A \cdot\left(\begin{array}{l}
d X_{0} \\
d Y_{0} \\
d Z_{0}
\end{array}\right)
$$

$$
d X_{0}=204,511083 \mathrm{~m}, d Y_{0}=42,192468 \mathrm{~m}, d Z_{0}=111,417880 \mathrm{~m} \text {. }
$$

In (Ha Minh Hoa, 2017) with purpose of comparision of an accuracy of series of the national quasigeoid heights $\zeta$ (1) with an accuracy of according series of the quasigeoid heights $\zeta^{*}(2)$ on the 164 GPS/first order levelling points, the both those series of the quasigeoid heights had been considered to be the equal accuracy at level of $\pm 0,062 \mathrm{~m}$. However, in practice the both above mentioned series of the quasigeoid heights don't have the same accuracy. In (Ha Minh while national quasigeoid heights $\zeta^{*}$ from the inital national quasigeoid model VIGAC2017 have been determined by following formula:

$$
\zeta^{*}=\overline{\bar{\zeta}}^{*}+A\left(\begin{array}{l}
d X_{0} \\
d Y_{0} \\
d Z_{0}
\end{array}\right),
$$

where the GPS/first and second orders levelling quasigeoid height $\zeta_{G P S}$ / leveling has been calculated by formula:

$$
\zeta_{\text {GPS / leveling }}=\bar{H}_{Z}-H_{Z}^{\gamma},
$$

$\bar{H}_{z}$ - geodetic height of the first (or second) order bench mark obtained from the GPS data processing in ITRF and converted to the zero - tide system; $H_{Z}^{\gamma}$ - first (or second) order national normal height converted to the zero - tide system; $\overline{\bar{\zeta}}^{*}$ - mixed quasigeoid height of point got from the mixed quasigeoid model VIGAC2014 and converted to the zero - tide system; matrix

$$
A=(\cos \bar{B} \cdot \cos \bar{L} \quad \cos \bar{B} \cdot \sin \bar{L} \quad \sin \bar{B}),
$$

$\bar{B}, \bar{L}$ - geodetic latitude and longitude of point according to the WGS84 ellipsoid; coordinate transformation parameters from ITRF to the VN2000-3D:

Hoa, 2017) RMS of the differencies $Z=\zeta-\zeta^{*}$ is equal to:

$m_{Z}= \pm \sqrt{m_{\zeta}^{2}+m_{\zeta^{*}}^{2}}= \pm \sqrt{\frac{\sum_{i=1}^{164} Z_{i}^{2}}{164}}= \pm \sqrt{\frac{1,265}{164}}= \pm 0,088 \mathrm{~m}$. Meanwhile in (Ha Minh Hoa et al., 2016) based on co - located GPS observations first order bench marks and global quassigeoid heights from the EGM2008 model on those bench marks. RMS of series of the quasigeoid 
heights $\zeta^{*}$ had been established at level of $m_{\zeta^{*}}= \pm 0,070 \mathrm{~m}$. When contribution portion of RMS $m_{\zeta}$ of series of the 164 national quasigeoid heights $\zeta$ to the RMS value $m_{Z}= \pm 0,088 \mathrm{~m}$ is equal to $\pm 0,053 \mathrm{~m}$.

As such for following usage in this article, we accept that the RMS of the national quasigeoid height $\zeta$ calculated by formula (1) from the corresponding GPS/first (or second) order levelling quasigeoid height $\zeta_{\text {GPS/leveling }}$ on the stable first (or second) order bench mark is equal to $\pm 0,053 \mathrm{~m}$, while the RMS of the national quasigeoid height $\zeta^{*}$ from the quasigeoid model VIGAC2017 calculated by formula (2) is equal to:

$$
m_{\zeta^{*}}= \pm 0,070 \mathrm{~m}
$$

With the purpose of improvement of accuracy of the quasigeoid model VIGAC2017 this scientific article will introduce results of fitting this model to the 194 GPS/first, second orders levelling quasigeoid heights by the least squares collocation.

\section{Data}

Apart from the 164 GPS/first, second orders leveling quasigeoid heights $\zeta$ for solving abovementioned task had been added 30 GPS/first order levelling quasigeoid heights in the zero - tide system on the stable first order bench marks obtained by Vietnam Institute of Geodesy and Cartography (VIGAC) in period 2012 - 2013 (Ha Minh Hoa, et al., 2012; Ha Minh Hoa, Nguyen Ba Thuy, Phan Trong Trinh, et al, 2016), Stability of the first order benchmarks had been controled by Smirnov's criteria (Smirnov N.V., Belugin D.A., 1969), The abovementioned 30 GPS/first order levelling quasigeoid heights had been converted to the national WGS84 reference ellipsoid by formula (1). On the 30 first order bench marks had been determined quasigeoid heights $\zeta^{*}$ according to the quasigeoid model VIGAC2017 by formula (2). The total 194 first and second orders bench marks have been distributed relatively regularly on whole territory of Vietnam.

\section{Applied methods}

We symbolize $\mathrm{Q}$ as a set of $\mathrm{n}$ GPS/first and second orders leveling bench marks (in our case $\mathrm{n}=194), \mathrm{P}$ as a set of points whose quasigeoid heights will be determined by the least squares collocation. In the set $Q$ had been calculated the differencies $Z_{i}=\zeta_{i}-\zeta_{i}^{*}, i=1,2, . ., 194$, where for point $\mathrm{i}$ the national quasigeoid height $\zeta_{i}$ had been determined by formula (1), while the quasigeoid height $\zeta_{i}^{*}$ from the quasigeoid model VIGAC2017 had been determined by formula (2). In addition the accuracy of the national quasigeoid height $\zeta_{i}$ is considered equal to $\pm 0,053 \mathrm{~m}$. On base of the least squares collocation, at a point $p \in P$, a national quasigeoid height $\tilde{\zeta}_{p}^{*}$ will be determined by formula:

$$
\tilde{\zeta}_{p}^{*}=\zeta_{p}^{*}+\delta \zeta_{p}^{*}
$$

where quasigeoid height $\zeta_{p}^{*}$ from the quasigeoid model VIGAC2017 is calculated by formula (2), correction $\delta \zeta_{p}^{*}$ is determined by formula (Moritz, H,, 1980):

$$
\delta \zeta_{p}^{*}=C_{p Q} \cdot K_{Z}^{-1} \cdot Z
$$

$$
C_{P Q}=\left(C_{p 1} C_{p 2} C_{p n}\right) \text { is the cross - }
$$
covariance matrix between the differences 
$Z_{i}=\zeta_{i}-\zeta_{i}^{*}(i=1,2, . ., 194)$, in the set $\mathrm{Q}$ and the estimated quasigeoid height at the point $p \in P, \mathrm{Z}$ is column - vector containing the differences $Z_{i}=\zeta_{i}-\zeta_{i}^{*}(i=1,2, . ., 194)$, covariance matrix has form:

$$
K_{Z}=C_{Z}+C_{Z Z} \text {, }
$$

$C_{Z}$ is the auto - covariance matrix of vector $\mathrm{Z}, C_{Z Z}$ is the covariance matrix, which reflects the spatial dependencies of the all differences $Z_{i}=\zeta_{i}-\zeta_{i}^{*}(i=1,2, . ., 194)$ in the set Q.

For the 194 differences

$Z_{i}=\zeta_{i}-\zeta_{i}^{*}(i=1,2, . ., 194)$, their $\quad$ RMS is equal to:

$$
m_{Z}= \pm \sqrt{\frac{1,580915}{194}}= \pm \sqrt{0,008149}=0,090 \mathrm{~m} .
$$

When the auto - covariance matrix $C_{Z}$ has the form:

$$
C_{Z}=m_{Z}^{2} \cdot E_{n x n}=0,008149 \cdot E_{n x n}<m^{2}>\text {, }
$$

where $E_{n x n}$ - unit matrix of order 194.

The covariance matrix $C_{Z Z}$, which reflects the spatial dependencies of the all differences $Z_{i}=\zeta_{i}-\zeta_{i}^{*}(i=1,2, . ., 194)$ in the set $\mathrm{Q}$, will be determined based on a covariance function

$$
C(d)=m_{Z}^{2}-\gamma(d)
$$

where $\gamma(d)$ is a semivariance; $d$ is a distance between any two points in the set $\mathrm{Q}$.

As such in our case the spatial dependence of quassigeoid heights in the set Q will be studied using semivariogram, The experimental semivariance $\gamma(h)$ at lag distance $\mathrm{h}$ is calculated by formula (Cressie N.A.C., 1993; Schabenger O., Gotway C.A., 2005; Marcin Ligas, Marek Kulczycki, 2014):

$$
\gamma(h)=\frac{1}{2 n_{h}} \cdot \sum_{i=1}^{n_{h}}\left(Z\left(x_{i}\right)-Z\left(x_{i}+h\right)\right)^{2},
$$

where $Z\left(x_{i}\right)$ is the difference $Z=\zeta-\zeta^{*}$ of the point at position $x_{i}, Z\left(x_{i}+h\right)$ is the difference $Z=\zeta-\zeta^{*}$ of the point at position $x_{i}+h$ separated from position $x_{i}$ by a distance not more than lag distance $h ; n_{h}$ is the number of pairs $Z\left(x_{i}\right)$.

By such way in the set $Q$ we must create groups of points, in addition in every group the distances between points not more than lag distance $h$. Based on an experimatal semivariogram we will determine form of theoretical semivariance, which in general case has following form:

$$
\gamma(d)=C_{0}+C_{1} \cdot f\left(\frac{d}{a}\right),
$$

where $C_{0}$ is the nugget effect; $C_{1}$ is the structural variance; $a$ is the range of spatial dependence; function $f\left(\frac{d}{a}\right)$ will be selected in relation to distribution of the semivariogram corresponding to standaed models of semivariance functions (Gaussian, spherical, exponential, linear models).

Value $C_{0}+C_{1}$ is the sill and determined from the semivariogram.

\section{Results}

From the 194 most stable co - located GPS observations first and second orders bench marks covering the whole territory of Vietnam had been constructed the set Q, which contains the 194 differences $Z=\zeta-\zeta^{*}$. In the set $Q$ had been created 58 groups of points with change of the distances from $25 \mathrm{~km}$ to $1475 \mathrm{~km}$. The lag distance $\mathrm{h}=25 \mathrm{~km}$.

For the semivariogram of the experimatal semivariances, shown in Figure 1, the sill 
$C_{0}+C_{1}=0,007928 \mathrm{~m}^{2}$, the range of spatial dependence $a=1475 \mathrm{~km}$. Next analysis results show that the nugget effect $C_{0}=0,002706 \mathrm{~m}^{2}$, the structural variance $C_{1}=0,005222 \mathrm{~m}^{2}$.
From the semivariogram of the experimatal semivariances we realize that distribution of the experimatal semivariances corresponds to spherical model. So the theoretical semivariance (10) has form:

$$
\gamma(d)=0,002706+0,005222 \cdot\left(\frac{3 \cdot d}{2 \cdot a}-\frac{1}{2} \cdot\left(\frac{d}{a}\right)^{3}\right)<m^{2}>.
$$

On account of the formulas (7), (11), the covariance function (8) gets form:

$$
C(d)=0,005443-0,005222 \cdot\left(\frac{3 \cdot d}{2 \cdot a}-\frac{1}{2} \cdot\left(\frac{d}{a}\right)^{3}\right)<m^{2}>\text {. }
$$

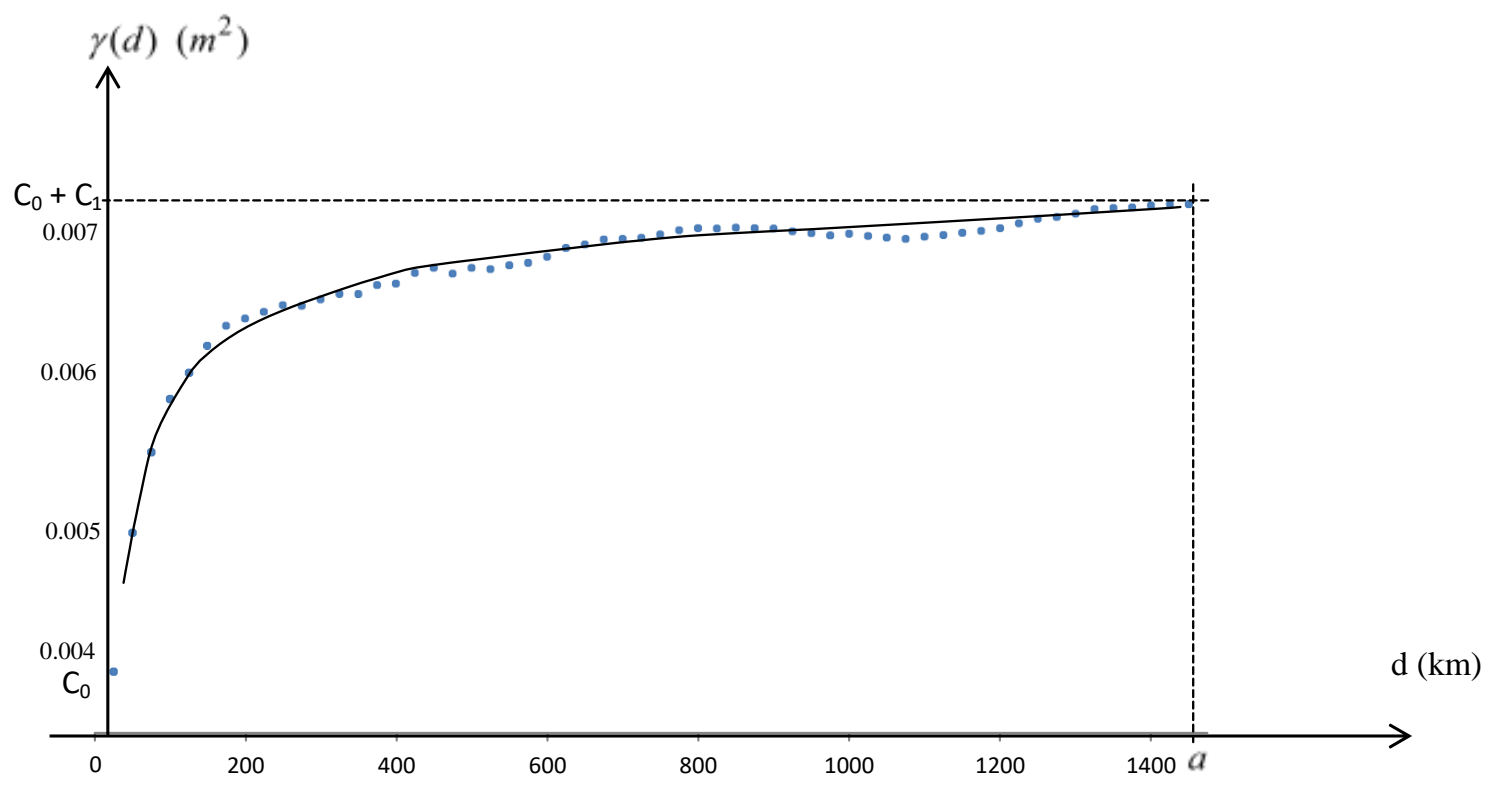

Figure 1. The semivariogram of the experimatal semivariances

After determination of the covariance matrix $C_{Z Z}$ baded on the the covariance function (12), on account of the auto covariance matrix $C_{Z}$ (8), we had calculated the covariance matrix $K_{Z}(6)$,

The correction $\delta \zeta_{p}^{*}$ to the quasigeoid height $\zeta_{p}^{*}$ of any point $p \in P$ was calculated by formula (5) and the corrected quasigeoid height $\tilde{\zeta}_{p}^{*}$ of this point was determined by formula (4). With purpose of accuracy estimation of the 194 corrected quasigeoid heights $\tilde{\zeta}^{*}$ of the quasigeoid model VIGAC2017 at the 194 first and second orders bench marks, we had calculated 194 differences $\bar{Z}_{i}=\zeta_{i}-\tilde{\zeta}_{i}^{*}(i=1,2, \ldots, 194)$, where $\zeta_{i}$ is the national quasigeoid height of bench mark $i$ calculated by formula (1) (see Table 1). 
Vietnam Journal of Earth Sciences, 40(1), 39-46

Table 1. The differences $\bar{Z}$ on the 194 first and second orders bench marks

\begin{tabular}{|c|c|c|c|c|c|c|c|c|}
\hline No & Points & $\begin{array}{c}\text { Differences } \\
\bar{Z}(\mathrm{~m})\end{array}$ & No & Points & $\begin{array}{c}\text { Differences } \\
\bar{Z}(\mathrm{~m})\end{array}$ & No & Points & $\begin{array}{c}\text { Differences } \\
\bar{Z} \text { (m) }\end{array}$ \\
\hline 1 & IBH-LS97 & 0,0543 & 66 & IVL-HT71 & 0,0523 & 131 & IILC-TG15 & 0,0427 \\
\hline 2 & IBH-TH122A & 0,0049 & 67 & IBH-TH59 & 0,0627 & 132 & IILC-TG19A & $-0,0469$ \\
\hline 3 & IBH-TH119 & 0,0246 & 68 & IVL-HT173-2 & 0,0860 & 133 & IILC-TG31 & 0,0422 \\
\hline 4 & IBH-HN33 & $-0,0141$ & 69 & IBH-TH70A & 0,0665 & 134 & IIMC-XM7-1 & $-0,0825$ \\
\hline 5 & IBH-HN39 & $-0,0123$ & 70 & IHN-VL50 & 0,1029 & 135 & IIMT-TH25 & $-0,1431$ \\
\hline 6 & IBH-HN42 & $-0,0410$ & 71 & IVL-HT123 & 0,0804 & 136 & IIMT-TH4 & $-0,0217$ \\
\hline 7 & IHN-HP7 & 0,0344 & 72 & ILS-HN12 & 0,0415 & 137 & IIMT-TH7 & $-0,1424$ \\
\hline 8 & IHN-VL10A & $-0,1006$ & 73 & IHP-MC4-1 & 0,0550 & 138 & IIMT-TV11 & $-0,0902$ \\
\hline 9 & IHN-VL4-1 & $-0,0039$ & 74 & IBH-LS80 & 0,0470 & 139 & IIMX-DC34 & $-0,1341$ \\
\hline 10 & IHN-VL6-1 & $-0,0206$ & 75 & IDN-BT86 & 0,0950 & 140 & IINB-HN11-1 & 0,0281 \\
\hline 11 & IDN-BMT16 & $-0,0646$ & 76 & IVL-HT320A & 0,1044 & 141 & IINB-HN15 & $-0,0019$ \\
\hline 12 & IDN-BMT28 & $-0,0582$ & 77 & IBMT-APD49-1 & 0,1158 & 142 & IINB-HN24 & 0,0397 \\
\hline 13 & IVL-HT150 & $-0,0686$ & 78 & IHP-NB14A & $-0,1340$ & 143 & IINB-HN27-1 & 0,0055 \\
\hline 14 & IVL-HT152-1 & $-0,0192$ & 79 & ILS-HN36 & 0,0140 & 144 & IINB-HN32-1 & 0,1176 \\
\hline 15 & IHN-VL34- & $-0,0504$ & 80 & ILS-HN22 & $-0,1483$ & 145 & IINK-PT10 & 0,0268 \\
\hline 16 & IHP-MC48A & $-0,0945$ & 81 & ILS-HN29 & $-0,0746$ & 146 & IINK-PT13 & 0,0887 \\
\hline 17 & IBH-TH3-1 & $-0,0572$ & 82 & IBH-HN16A & 0,0509 & 147 & IINK-PT6-1 & $-0,2096$ \\
\hline 18 & IVL-HT181 & $-0,0485$ & 83 & IHN-VL28-1 & 0,0222 & 148 & IIPLK-PL12 & $-0,0317$ \\
\hline 19 & ILS-TY4 & $-0,0933$ & 84 & IBH-HN48 & 0,0954 & 149 & IIPLK-PL16 & $-0,0667$ \\
\hline 20 & IVL-HT309A & $-0,0278$ & 85 & IHN-HP2A & 0,0859 & 150 & IIPLK-PL2 & 0,0641 \\
\hline 21 & IVL-HT317 & $-0,0323$ & 86 & IHN-HP5 & 0,1210 & 151 & IIPLK-PL24 & $-0,1687$ \\
\hline 22 & IVL-HT187 & $-0,0337$ & 87 & IVL-HT73 & 0,1703 & 152 & IIPLK-PL8 & $-0,0346$ \\
\hline 23 & IVL-HT170-1 & $-0,0414$ & 88 & IVL-HT95 & 0,1522 & 153 & IISC-PL29 & $-0,0922$ \\
\hline 24 & IHP-MC41 & $-0,0684$ & 89 & IIDK-TM41 & 0,0320 & 154 & IISC-VT3-1 & 0,0001 \\
\hline 25 & IHN-VL56 & 0,0631 & 90 & IIAB-CL5 & $-0,0628$ & 155 & IITL-TV5-1 & $-0,0861$ \\
\hline 26 & IBH-TH11 & 0,0272 & 91 & IIAS-KS10 & $-0,1188$ & 156 & IITL-TV7 & $-0,0792$ \\
\hline 27 & IHN-VL40-1 & 0,0619 & 92 & IIAS-KS16 & $-0,0715$ & 157 & IITT-TK29 & $-0,1479$ \\
\hline 28 & IVL-HT130 & $-0,0353$ & 93 & IIAS-KS22 & $-0,1120$ & 158 & IITX-TL14 & $-0,0624$ \\
\hline 29 & IBH-LS77 & 0,0036 & 94 & IIAS-KS32 & $-0,0971$ & 159 & IITX-TL20-1 & $-0,0886$ \\
\hline 30 & IBH-TH5 & $-0,0512$ & 95 & IIAS-KS35 & $-0,1490$ & 160 & IITX-TL25 & $-0,0068$ \\
\hline 31 & IHN-VL38-1 & $-0,0157$ & 96 & IIBH-XL11-1 & $-0,0204$ & 161 & IITX-TL6 & $-0,0214$ \\
\hline 32 & IVL-HT197 & $-0,0177$ & 97 & IIBH-XL17 & 0,0250 & 162 & IIYB-CN18 & $-0,0811$ \\
\hline 33 & IBMT-APD63 & $-0,0186$ & 98 & IIBH-XL6 & 0,1134 & 163 & IIYB-CN24-1 & $-0,1574$ \\
\hline 34 & IVL-HT127-3 & $-0,0283$ & 99 & IIBMT-DT12 & $-0,0944$ & 164 & IDN-BT18-1 & $-0,0764$ \\
\hline 35 & IBMT-APD59-1 & $-0,0199$ & 100 & IIBMT-DT14 & $-0,1441$ & 165 & IBMT-APD46 & $-0,0854$ \\
\hline 36 & IVL-HT278-1 & 0,0208 & 101 & IIBMT-DT4 & 0,1568 & 166 & IVL-HT305 & $-0,0510$ \\
\hline 37 & IVL-HT108 & $-0,0264$ & 102 & IIBN-QT11-1 & 0,1120 & 167 & IVL-HT159-3 & 0,1423 \\
\hline 38 & IDN-BT77 & $-0,0083$ & 103 & IIBS-CD12 & $-0,0333$ & 168 & IVL-HT262A & 0,1721 \\
\hline 39 & IBMT-NH17-1 & $-0,0103$ & 104 & IIBS-CD14 & 0,1611 & 169 & IHN-VL76 & 0,1302 \\
\hline 40 & IVL-HT83 & $-0,0326$ & 105 & IIBS-CD3 & 0,0155 & 170 & IVL-HT113 & 0,1196 \\
\hline 41 & IBH-HN17 & $-0,0392$ & 106 & IIBS-CD7-1 & 0,0832 & 171 & ILS-HN10 & 0,0748 \\
\hline 42 & IHN-VL45-1 & 0,0611 & 107 & IICD-HN6 & 0,1058 & 172 & IBH-HN19-1 & 0,1009 \\
\hline 43 & IBH-TH65 & $-0,0178$ & 108 & IICD-VC4 & $-0,1091$ & 173 & IBMT-NH11-1 & 0,1350 \\
\hline 44 & IVL-HT178 & 0,0113 & 109 & IICD-VC4-1 & 0,0054 & 174 & IBH-HN20-1 & 0,1026 \\
\hline 45 & IVL-HT103 & $-0,0079$ & 110 & IICT-GD1 & 0,1305 & 175 & TB01 & 0,1079 \\
\hline 46 & IHN-VL64 & 0,0259 & 111 & IICT-GD10 & 0,0103 & 176 & QN01 & $-0,0246$ \\
\hline 47 & IVL-HT141-3 & 0,0082 & 112 & IICT-GD15-1 & $-0,0216$ & 177 & QNG1 & $-0,1084$ \\
\hline 48 & IVL-HT329A & 0,0175 & 113 & IICT-GD4 & 0,1442 & 178 & ВР01 & 0,0219 \\
\hline 49 & IHN-VL72 & 0,0225 & 114 & IICF-VT1 & 0,0049 & 179 & 22A1 & $-0,0264$ \\
\hline 50 & IVL-HT158 & 0,0264 & 115 & IIDK-TM29 & $-0,0886$ & 180 & 38A1 & $-0,0757$ \\
\hline 51 & IVL-HT121 & 0,0765 & 116 & IIDK-TM45 & $-0,1262$ & 181 & VL48 & 0,0401 \\
\hline 52 & IDN-BT74 & 0,0485 & 117 & IIDL-PR31 & $-0,1293$ & 182 & IHN-VL59 & 0,0123 \\
\hline
\end{tabular}


Ha Minh Hoa/Vietnam Journal of Earth Sciences 40 (2018)

\begin{tabular}{llclcll}
\hline 53 & IBH-LS88-1 & $-0,0155$ & 118 IIGD-AB12 & $-0,0212$ & 183 VL73 & 0,1348 \\
54 & IVL-HT98 & 0,0110 & 119 IIGD-AB3-1 & $-0,0451$ & 184 HT73 & 0,1263 \\
55 & IBH-LS85-1 & $-0,0117$ & 120 IIGD-AB9-1 & $-0,0068$ & 185 HT84 & 0,0415 \\
56 & IBH-LS93 & $-0,0133$ & 121 IIGD-APD2-1 & 0,1062 & 186 HT94 & 0,0882 \\
57 & IBH-LS71 & $-0,0074$ & 122 IIGD-APD6-1 & $-0,0193$ & 187 HT106 & 0,0137 \\
58 & IBT-APD56 & 0,0382 & 123 IIHN-AB11 & $-0,0317$ & 188 HT121 & $-0,0415$ \\
59 & IVL-HT87 & 0,0281 & 124 IIHN-AB17 & $-0,0880$ & 189 HT127-4 & 0,0117 \\
60 & IVL-HT247A & 0,0574 & 125 IIHN-AB20 & $-0,0542$ & 190 IVL-HT141-3 & 0,0622 \\
61 & ILS-TY1 & 0,0040 & 126 IIHN-AB23 & $-0,0333$ & 191 HT159-1 & $-0,0326$ \\
62 & IVL-HT325-1 & 0,1074 & 127 IIHN-AB3 & $-0,0346$ & 192 HT173-3 & $-0,0243$ \\
63 & IDN-BT83 & 0,0552 & 128 IIHN-AB7 & $-0,1025$ & 193 HT197 & 0,0932 \\
64 & IVL-HT78 & 0,0298 & 129 IIHN-MT15 & $-0,0598$ & 194 IHP-MC45 & 0,0950 \\
65 & ILS-HN7 & 0,0170 & 130 IIHN-MT5 & 0,0092 & & \\
\hline
\end{tabular}

The RMS of the differences $\bar{Z}_{i}=\zeta_{i}-\tilde{\zeta}_{i}^{*}(i=1,2, \ldots, 194)$ is equal to:

$m_{\bar{Z}}= \pm \sqrt{\frac{\sum_{i=1}^{194} \bar{Z}_{i}^{2}}{194}}= \pm \sqrt{\frac{1,1750}{194}}= \pm 0,078 \mathrm{~m}$.

Because the RMS of the national quasigeoid heights $\zeta$ calculated by formula (1) got equal to $m_{\zeta}= \pm 0,053 m$, the contribution portion of RMS $m_{\tilde{\zeta}^{*}}$ of the quasigeoid heights $\tilde{\zeta}^{*}$ of the corrected quasigeoid model VIGAC2017 to the RMS value $m_{\bar{Z}}= \pm 0,078 m$ is equal to $\pm 0,058 \mathrm{~m}$.

From the RMS values $m_{\tilde{\zeta}^{*}}= \pm 0,058 \mathrm{~m}$ and $m_{\zeta^{*}}$ (3) we realize that in comparison with the initial quasigeoid model VIGAC2017, the corrected quasigeoid model VIGAC2017 has been more accurate than $20,69 \%$.

\section{Discussions}

Research results show that after fitting the initial quasigeoid model VIGAC2017 to 194 national quasigeoid heights at the first and second orders bench marks by the least squares collocation, accuracy of the corrected quasigeoid model VIGAC2017 had been increased to $20,69 \%$. That has been obtained taking into account the spatial dependences of the quasigeoid heights in the Earth gravity field on territory of Vietnam.

However, the corrected quasigeoid model VIGAC2017 still does not obtain accuracy more than $4 \mathrm{~cm}$. The next increase of accuracy of the national quasigeoid model in Vietnam will be accomplished in the future on base of using detailed gravimetric data.

\section{Conclusions}

Above represented research results show, that on the base of solving the task of fitting the initial quasigeoid model VIGAC2017 to the 194 national quasigeoid heights got from the 194 GPS/first and second orders levelling quasigeoid heights by the least squares collocation, the accuracy of the this model has been increased to to $20,69 \%$. That had been obtained due to taking into account the spatial dependences of the quasigeoid heights in the Earth gravity field on territory of Vietnam, With obtained accuracy of $\pm 0,058 \mathrm{~m}$ the corrected quasigeoid model VIGAC2017 may be used for solving of some tasks related to physical geodesy in the initial spatial reference system VN2000-3D.

A perfection of the national spatial reference system in relation to step by step accuracy improvement of the national quasigeoid model is iterative process. After accomplishment of detailed gravimetric measurements on whole territory of Vietnam 
Ha Minh Hoa/Vietnam Journal of Earth Sciences 40 (2018)

will be realized the next accuracy improvement of the national quasigeoid model, That will create conditions for the next perfection of the national spatial reference system in Vietnam in the future.

\section{References}

Cressie N.A.C., 1993. Statistics for spatial data, John Wiley \& Sons. New York, 900p.

Ha Minh Hoa, et al., 2012. Research scientific base for perfection of the height system in connection with construction of national dynamic reference system. General report of the science - technological teme of the Ministry of Natural Resources and Environment, Hanoi, 247p.

Ha Minh Hoa (Editor), 2016. Research for determination of normal surfaces of sea levels ("zero" depth surface, mean sea surface, highest sea surface) by methods of geodesy, hydrography and geology with serving construction of buildings and planning of coastline in tendency of climate changes”. State techno - scientific theme with code KC.09.19/11-15 in period of 2011-2015, Vietnam Ministry of Science and Technology, Hanoi, 563p.

Huang J., Véronneau M., 2013. Contribution of the GRACE and GOCE models to a geopotential based geodetich vertical datum in Canada. Geophysical Research Abstracts, 15, EGU201310164.
Iliffe J.C., Ziebart M., Cross P.A., Forsberg R., Strykowski G., Tscherning C.C., 2003. OSGM02: A New model for converting GPS-derived heights to local height datums in Great Britain and Ireland. Survey Review, 37(290), 276-293.

Marcin Ligas, Marek Kulczycki, 2014. Kriging approch for local height transformations. J, Geodesy And Cartography, Polish Academy of Sciences, 63(1), 537, Doi: 10,2478/geocart-2014-0002.

Metin Soycan, 2014. Improving EGM2008 by GPS and leveling data at local scale. BCG - Boletin de Ciências Geodésicas Sec, Artigos, Curitiba, 20(1), 3-18, on - lineversion, ISSN 1982-2170, Doi,org/10,1590/S1982-21702014000100001.

Moritz H., 1980. Advanced Physical Geodesy. Herbert wichmann Verlag Karlsruhe, Abacus Press Tunbridge Wells Ken, 512p.

Quasigeoid of the Federal Republic of Germany GCG2016. Federal Agency for Cartography and Geodesy, www,geodatenzentrum.de.

Roman D.R., Wang Y.M., Saleh J., Li X., 2010. Geodesy, Geoids \& Vertical Datums: A Perspective from the U,S, National Geodetic Survey. FIG Congress 2010, Sydney, Australia, April 2010, 11-16.

Schabenger O., Gotway C.A., 2005. Statistical methods for spatial data analysis. Chapman \& Hall/CRC, New York, ISBN 1-58488-322-7, 488p.

Smirnov N.V., Belugin D.A., 1969. Probability theory and mathematical statistics in applying to geodesy. Moscow, Nedra, 379p. 